\title{
Use of the Humeral Head as a Reference Point to Prevent Axillary Nerve Damage during Proximal Fixation of Humeral Fractures: An Anatomical and Radiographic Study
}

\author{
Theeuwes $\mathrm{HP}^{1}$, Potters $\mathrm{JW}^{2}$, Bessems $\mathrm{JHJM}^{3}$, Kerver $\mathrm{AJ}^{4}$, Kleinrensink GJ ${ }^{5}$
}

\begin{abstract}
Introduction: Treatment of proximal humeral fractures with plate osteosynthesis or intramedullary nail fixation in humeral shaft fractures with a proximal locking bolt carries the risk of iatrogenic injury of the axillary nerve. The purpose of this anatomical study is to define a more reliable safe zone to prevent iatrogenic axillary nerve injury using the humeral head instead of the acromion as a (radiographic) reference point during operative treatment.

Materials and methods: Anatomical dissection and labeling of the axillary nerve and branches was performed on 10 specially embalmed human specimens. Standard AP and straight lateral radiographs were made. The distances were measured indirectly from the cranial tip of the humerus to the axillary nerve on radiographs.

Results: The median distance from the cranial tip of the humerus to the axillary nerve was $52 \mathrm{~mm}$. The mean number of axillary nerve branches was 3. The distances from the cranial tip of the humerus to the nerve (branch) varied from 23 to $78 \mathrm{~mm}$. The median distance from the proximal (anterior) branch was $36 \mathrm{~mm}$, to the second branch $47 \mathrm{~mm}, 54 \mathrm{~mm}$ to the third branch and $73 \mathrm{~mm}$ to the fourth branch. The axillary nerve moves along with the humerus in cranial and caudal direction when the subacromial space varies.

Conclusion: This study shows that the position of the axillary nerve can be better determent using the cranial tip of the humerus as a reference point instead of the acromion. Furthermore, it is unsafe to place the proximal locking bolts in the zone between $24 \mathrm{~mm}$ and $78 \mathrm{~mm}$ from the cranial tip of the humerus. The greatest chance to cause a lesion of the main branch of the axillary nerve is in the zone between $48 \mathrm{~mm}$ and 58 $\mathrm{mm}$ caudal from the tip of the humeral head.

Keywords: Axillary nerve damage, Proximal humeral fracture, Quadrilateral space, Radiography, Safe zone, Surgical anatomy, Trauma surgery. Strategies in Trauma and Limb Reconstruction (2020): 10.5005/jp-journals-10080-1460
\end{abstract}

\section{INTRODUCTION}

Proximal humerus fractures account for between $5 \%$ and $6 \%$ of all fractures. ${ }^{1}$ Displaced and non-displaced fractures of the proximal humerus involving the surgical neck are mainly treated conservatively. ${ }^{2}$ However, open fractures and fractures involving neurovascular injury are strong indications for surgical intervention. The most frequently used operative techniques for proximal humeral fractures (PHF) are plate osteosynthesis (open deltopectoral and deltoid-split approaches) and intramedullary nail fixation with proximal and distal locking screws. For humeral shaft fractures (HSF), nailing is becoming more popular. ${ }^{3}$ However, all operative techniques carry the risk of iatrogenic injury of the proximal axillary nerve or the distal radial nerve, both in the anterior and the lateral humeral approaches. ${ }^{4,5}$ Damage to the axillary nerve can be inflicted before, during or after surgical intervention. ${ }^{6}$

Numerous papers have been published that describe the course of this nerve and define an anatomical safe zone for the axillary nerve during surgical dissection of the deltoid muscle, thereby preventing iatrogenic axillary nerve damage. ${ }^{6-11}$ Most of these studies focused on the main branch of the axillary nerve (anterior and posterior) and its position in relation to the acromion. Other studies describe the branching of the posterior branch in the deltoid muscle. ${ }^{12-14}$ However, as far as we are aware, the studies that defined anatomical safe zones for shoulder surgery took no account of radiographic parameters, which we think, are of added value in clinical situations. The aim of this
${ }^{1}$ Department of Surgery, Elisabeth-TweeSteden Hospital, Tilburg, The Netherlands; Department of Neuroscience, Erasmus MC Anatomy and Research Program, Rotterdam, The Netherlands

2,3 Department of Anesthesiology, Medisch Spectrum Twente, Enschede, The Netherlands; Department of Neuroscience, Erasmus MC Anatomy and Research Program, Rotterdam, The Netherlands

${ }^{4}$ Department of Surgery, Franciscus Gasthuis and Vlietland, Rotterdam, The Netherlands; Department of Neuroscience, Erasmus MC Anatomy and Research Program, Rotterdam, The Netherlands

${ }^{5}$ Department of Neuroscience, Erasmus MC Anatomy and Research Program, Rotterdam, The Netherlands

Corresponding Author: Theeuwes HP, Department of Surgery, Elisabeth-TweeSteden Hospital, Tilburg, The Netherlands; Department of Neuroscience, Erasmus MC Anatomy and Research Program, Rotterdam, The Netherlands, Phone: +31-6-18112401, e-mail: hilcotheeuwes@gmail.com

How to cite this article: Theeuwes HP, Potters JW, Bessems JHJM, et al. Use of the Humeral Head as a Reference Point to Prevent Axillary Nerve Damage during Proximal Fixation of Humeral Fractures: An Anatomical and Radiographic Study. Strategies Trauma Limb Reconstr 2020;15(2):63-68.

Source of support: Nil

Conflict of interest: None

study was therefore to provide important additional information for surgical interventions involving the proximal humerus by combining anatomical and radiological parameters. We wanted 
to define a more specific safe zone and to improve awareness of the potential position of the axillary nerve, especially the positions of the distal branches that run into the deltoid muscle. Our combined anatomical and radiological approach allowed us to also study the position of the anterior branch of the axillary nerve in relation to the acromion and deltoid muscle after the nerve leaves the quadrilateral space. We also provide a diagram showing the dynamic position of the motor branches of the axillary nerve during caudal force on the arm. The resulting increase in the distance between the acromion and the axillary is clinically relevant during drilling and blunt insertion of proximal bolts while performing a deltoid split. We thereby introduce the concept of a "dynamic safe zone".

\section{Materials and Methods}

Ten flexible embalmed cadavers of Caucasian adults were used in this study, providing us with 20 shoulders, 10 left and 10 right. Seven cadavers were female and three male, and age ranged between 40 years and 89 years. We had no further information regarding previous medical history, not provided in the Netherlands.

AnubifiX $\mathrm{X}^{\oplus}$ embalming was performed at room temperature between 24 hours and 72 hours postmortem. In human specimens prepared with this new embalming method, movements of the joints and tissues mimic those in "real life", thereby avoiding the stiffness and deterioration seen in specimens prepared with normal embalming methods and allowing for use in clinically relevant research and practice. ${ }^{15-17}$

\section{Anatomical Definitions}

The axillary nerve has both sensory and motor functions, with the motor function split between the anterior and the posterior terminal branches. The anterior branch ascends around the surgical neck of the humerus, accompanying the posterior circumflex humeral artery and its branches are distributed anteriorly into the deltoid muscle. Here, it innervates the entire anterior (clavicular) and middle (acromial) part of the deltoid muscle, and in most cases $(91.5 \%)$, it also innervates the posterior (scapular spine) portion. The three subdivisions of the deltoid muscle also have three different insertions (anterior, middle and posterior) on the humeral shaft, with an average insertion length of approximately $39 \mathrm{~mm} .{ }^{18-20}$ The posterior branch of the axillary nerve only innervates the posterior part of the deltoid in $97.7 \%$ of the cases. ${ }^{14,21}$ It also innervates the long head of the triceps brachii and teres minor muscle. ${ }^{22,23}$

\section{Dissection and Labelling}

Prior to radiography, the humerus was dissected and labelled using the following procedure: The skin of the proximal part of the humerus was first dissected. The ventral origin of the deltoid muscle was then detached from the clavicle and acromion by sharp dissection using a deltopectoral approach. A part of the deltoid muscle was cut distally at the point of insertion to expose the humeral (inner) side of the muscle (Fig. 1). The anterior branch of the axillary nerve and distal (motor) branches were then identified. The course of the axillary nerve and its branches was marked with radio-opaque wire. All wires were fixed onto the nerves using metal clips. To identify the different (sub)branches, the anterior, middle and posterior segments of the deltoid muscle were marked with $1,2,3$ or 4 clips; the anterior (main) branch was marked with a larger diameter wire as illustrated in Figures 1 and 2. After placing

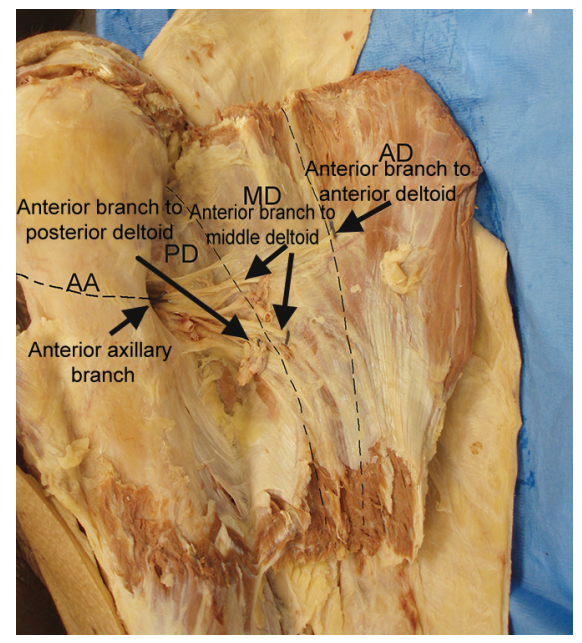

Fig. 1: Anatomical dissection of a left deltoid muscle and labelling with radiopaque wires and clips prior to radiography

the markers, the deltoid muscle was reattached in its anatomical position with sutures.

\section{Measurements}

Radiographs were obtained with a mobile C-arm system (Philips). Radiographs were taken in an anteroposterior direction, with the humerus in neutral position, the forearm resting on a support and the elbow flexed at $90^{\circ}$. To take true lateral (perpendicular) images, we then removed the arms from the torsos approximately $8 \mathrm{~cm}$ medial to the glenoid, respecting the integrity of the attached muscles and neurovascular structures. Such lateral images show the true position of the axillary nerve and its branches during surgical procedures as seen from a lateral position. To guarantee a true lateral image, a Kirschner wire was positioned in the head of the humerus, in line with the medial and lateral epicondyle projection on X-ray imaging. Image analysis was performed directly on the radiographic image using ImageJ software (v1.41/1.51f) ${ }^{24}$ as demonstrated in Figure 2. In order to correct for magnification errors, a calibration bar was visible on all images and used for rescaling. The position of the anterior branch of the axillary nerve was determined by measuring the distance between its intersection with the middle line of the humerus and the cranial edge of the humeral head (blue lines in Fig. 2). The distal (motor) branches innervating the anterior, middle and posterior part of the deltoid muscle were measured from the top of the humerus to the clip(s), marking the entry point into the deltoid muscle (yellow lines in Fig. 2).

All images and measurements obtained in the above experiments were taken in a static situation. After analysing our data, we performed an extra pilot experiment to analyse the dynamic situation of the axillary nerve. To this end, we labelled the main branch and applied a 50-Newton traction force on the humerus in a downward direction taking radiographic images before and after traction.

\section{Results}

To explore variations in the anatomical course of the axillary nerve, we analysed images from 20 upper arms. The mean number of axillary nerve branches to the deltoid muscle was 3 . The mean 


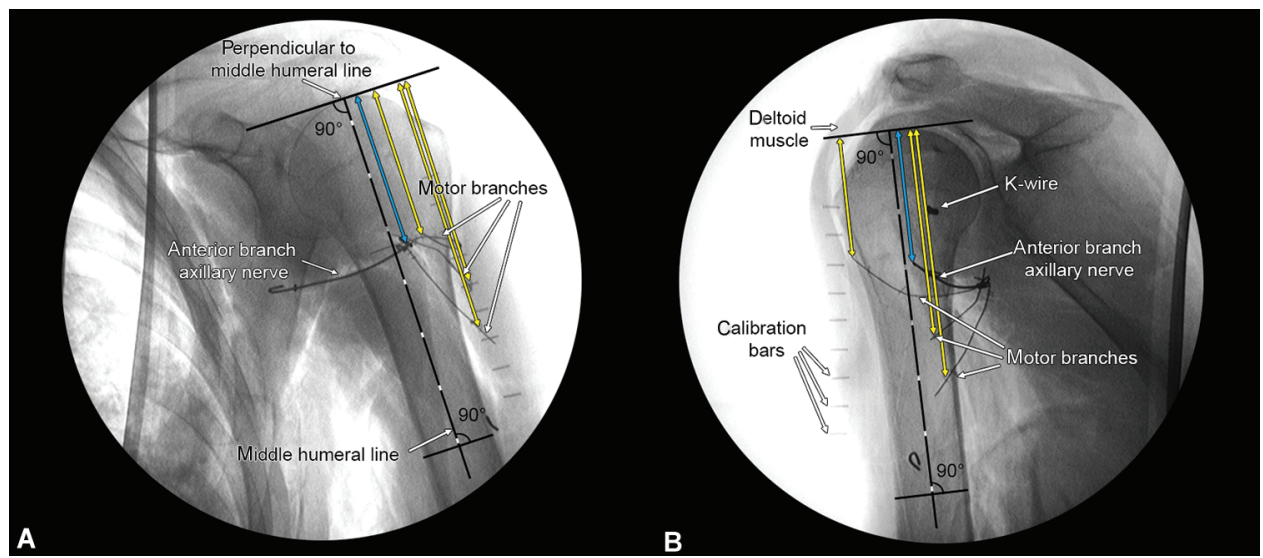

Figs $2 \mathrm{~A}$ and B: Measurement methods on radiographs. (A) Anteroposterior view of a left humerus; (B) A true lateral radiograph obtained after removing the same left shoulder from the torso

Table 1: Mean distances (in $\mathrm{cm}$ ) from cranial edge of the humeral head to the axillary nerve and its branches (AD, anterior deltoid muscle; $M D$, middle deltoid muscle; PD, posterior deltoid muscle)

\begin{tabular}{lllll}
\hline Axillary branch & Position X-ray & $\begin{array}{l}\text { Mean } \\
\text { distance }(\mathrm{cm})\end{array}$ & SD & $n$ \\
\hline Main & Anteroposterior & $5.8(4.8-7.5)$ & 0.7 & 20 \\
& Lateromedial & $6.3(4.7-7.9)$ & 1 & 10 \\
AD (anterior) & Lateromedial & $5.3(4.3-6.1)$ & 0.7 & 10 \\
MD (middle) & Lateromedial & $7.2(6.7-7.6)$ & 0.3 & 5 \\
PD (distal) & Lateromedial & $8.8(7.1-9.7)$ & 0.8 & 7 \\
\hline
\end{tabular}

distance of the (main) anterior axillary branch from the top of the humeral head in lateral view was $6.3 \mathrm{~cm}$ (range 4.7-7.9); in anteroposterior view $5.8 \mathrm{~cm}$ (range 4.8-7.5). The branches into the anterior deltoid part (AD), middle deltoid part (MD) and posterior deltoid part were as follows. AD; in lateral view, was $5.3 \mathrm{~cm}$ (range 4.3-6.1), MD $7.2 \mathrm{~cm}$ (range 6.7-7.6) to the middle branch while that to the PD was $8.8 \mathrm{~cm}$ (range 7.1-9.7) (Table 1). Figure 3 illustrates the position of the axillary nerve and its branches in relation to the humerus. It also illustrates the zones that are relatively safe for anterior and lateral approaches to the proximal humerus (green areas).

The results of our additional pilot experiment are shown in Figure 4. Figure $4 A$ shows an anteroposterior radiograph with the upper arm in neutral position and no downward traction. Figure 4B shows the same upper arm with 50-Newton traction force. Line 1 indicates the height of the subacromial space $(0.9 \mathrm{~mm})$, with line $1^{\prime}$ in Figure $4 \mathrm{~B}$ indicating the same distance during traction. The white line marked with a plus sign (+) in Figure $4 \mathrm{~B}$ indicates the increase in the height of the subacromial space (also $0.9 \mathrm{~mm}$ ). Lines 2, 3 and 4 have been copied from Figure $4 \mathrm{~A}$ and transferred to Figure $4 \mathrm{~B}$ to show the equivalent distance from the humeral head to the axillary nerve in a neutral position. The white lines indicate increases in the distance from the acromion to the axillary nerve, at different positions along the axis of rotation in a medial to lateral direction: Increased distances of $0.3,0.4$ and $0.8 \mathrm{~mm}$. This demonstrates that the position of the axillary nerve moves like an arm ( $r$; radius) around the axis of rotation (entry of axillary nerve from quadrilateral foramen). During downward traction, the angle of rotation increases 8 degrees from 63 to $71^{\circ}$ rotation in this additional pilot experiment.
The more lateral the axillary nerve (i.e., the further away from the axis point), the greater the distance between the acromion and the axillary nerve becomes.

Although the distance from the top of the humeral head to the axillary nerve differs as well, it seems to remain relatively constant on the lateral border of the humerus on this radiograph (see dark green line labelled 4 " which is a copy of the 4 ' from Figure 4A).

\section{Discussion}

Based on the results of our static experiments, we suggest that surgeons should be particularly careful while drilling in an anterior to posterior direction, at a distance between 4.8 and $7.8 \mathrm{~cm}$ distal to the top of the humeral head. This finding is in line with reports in the literature showing that the anterior branch of the axillary nerve curves very tightly around the back of the humerus, making it especially vulnerable to iatrogenic damage. ${ }^{9,25,26}$ The anterior axillary branch and its sub-branches may extend from $4.3 \mathrm{~cm}$ in lateral view to $9.7 \mathrm{~cm}$ distal to the top of the humeral head. This making it more likely that an approach from the lateral side will inflict damage to one of the branches of the axillary nerve.

Here, we have shown that the anterior branch of the axillary nerve, which innervates the deltoid muscle, has an anatomical course that varies among Caucasian people. Our anatomical results were restricted to specimens of Caucasian origin; extrapolation to other ethnic groups requires further investigation of other specific ethnic groups. Any damage to the axillary nerve (anterior branch) can significantly affect the flexor and abductor function of the patient's arm, and therefore most of its extension. As a consequence, the surgeon must be cautious to avoid neurotmesis of the axillary nerve and its distal (motor) branches. Such damage can occur during nail fixation of the proximal humerus with proximal screw or blade fixation. The potential risk of axillary nerve damage while using different humeral nails is well illustrated in a study by Nijs et al. ${ }^{27}$

Nerve damage is also possible while performing plate osteosynthesis with an open deltoid-split or minimally invasive deltoid-splitting approach. During such procedures, surgeons should consider the location of the axillary nerve where it curves around the humerus from medial to lateral via posterior and divides into three branches, as illustrated in Figure 3. 
Anterior view

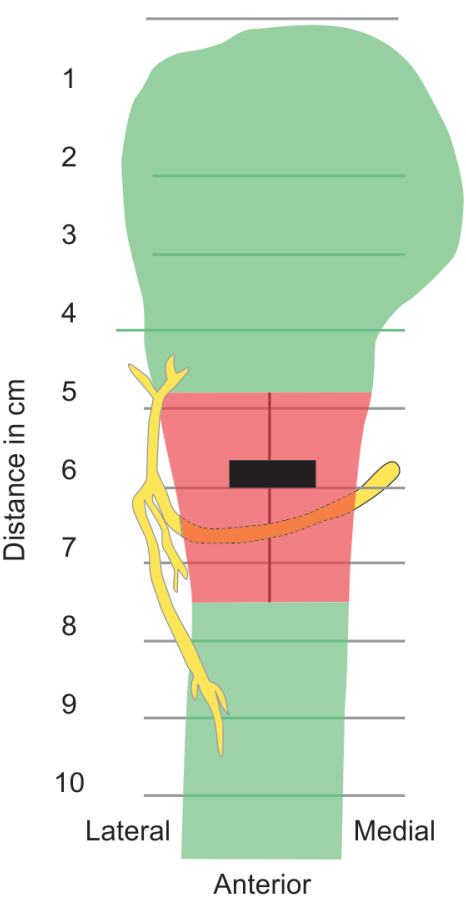

and distal branches

(behind humerus)
Lateral view

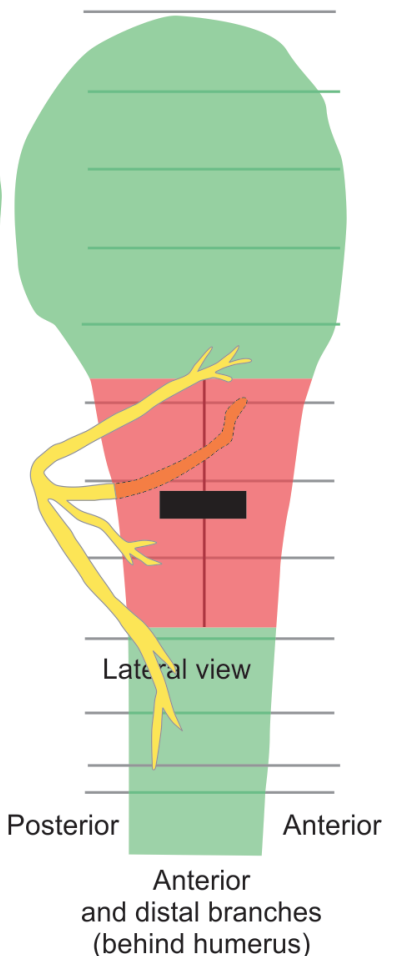

Lateral view

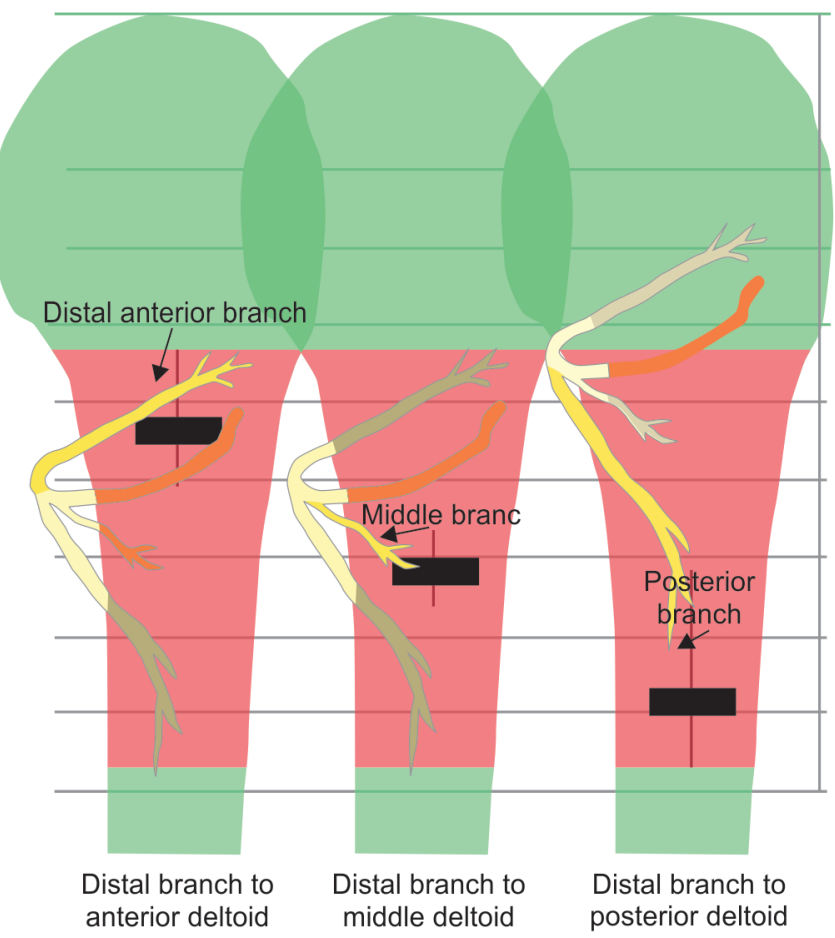

Fig. 3: Safe zones for axillary nerve and branches in terms of distance in centimetres from the top of the humerus in a static situation. Red indicates areas in which there is a danger of damaging the anterior branch of the axillary nerve while penetrating the humerus from an anterior or lateral position. Green indicates relatively safe areas for placing locking screws and deltoid splitting (proximal) in anterolateral approaches



Figs 4A and B: Right shoulder showing the increase in the size of the subacromial space and the change in the relative position of the anterior branch of the axillary nerve around the axis of rotation. (A) Normal position; (B) During downward caudal traction with 50-Newton force. See main text for explanation of different colours, numbers and letters

Since the middle branch innervates the clavicular (lateral) part of the deltoid muscle, it is clinically the most at risk of damage during a lateral approach (deltoid split). latrogenic injury of this nerve branch results in sensory loss, muscle atrophy and muscle weakness.

During a lateral approach, the main axillary branch is also at potential risk of damage, e.g., while drilling through the medial cortex, from lateral to medial, even though the main nerve axillary is not fixed to the humerus in this location.

We chose to measure the distance of the axillary nerve from the top of the humeral head rather than from the acromial edge for several reasons. First, the distance between the humerus and the acromion (subacromial space) can vary due to traumatically induced haemarthrosis, dislocation of the fracture parts, trauma- 
induced relaxation or even spasm of the deltoid muscle (Fig. 4). Vathana et al. have previously described the natural variety in the relative positions of the acromion and the axillary nerve, and suggest that this a good reason why the acromion should not be used as a reference point. ${ }^{28}$

A second reason for not using the acromion as a reference point is that the natural anatomical shape of the acromion differs among from person to person. ${ }^{29}$ Cheung et al. analysed the dynamic position of the axillary nerve in relation to the acromion and showed that a vertical abduction of $60^{\circ}$ in the glenohumeral joint increased the distance of the axillary nerve to the acromion; although they did not report on the position after applying a traction force to the upper arm. ${ }^{30}$

In this study, we have tried to relate the course of the axillary nerve and its branches to clinically relevant situations during shoulder interventions, i.e., the anterior and lateral surgical approaches to the proximal humerus. Our measurements reflect a relatively static situation in which the data can be compared with other data that have been published on the course of the axillary nerve. Our static data are complemented by the data from our pilot experiment in which we applied a downward traction force to the cadaveric arm to mimic the clinical situation during general anesthesia and muscle relaxation. The variability that we observed in the height of the subacromial space and in the course of the anterior branch of the axillary nerve, as illustrated in Figure 4, suggests that such measurements are dynamic rather than static. In Figure 5, we tried to illustrate the relatively fixed position of the anterior branch of the axillary nerve to the lateral side of the humerus, with and without connective tissue: The nerve curves around the surgical neck on the posterior side of the humerus shaft. The effect of caudally directed traction on the humerus is shown in relation to the position of the axillary nerve in Figure 4. The anterior branch of the axillary nerve moves downward with the humerus, staying in a position that is relatively fixed in relation to the cranial top of the humerus (lateral side), as indicated by the dark green line marked 4". However, the position of the axillary nerve in relation to the acromion changes when caudally directed traction is applied, as indicated by the white arrows in Figure 4B.

The movement of the anterior branch of the axillary nerve can only partly be explained by its "fixation" to the posterolateral part of the humerus after it leaves the quadrilateral space. The nerve appears to move around a fixed axis point, as shown in Figure 4.

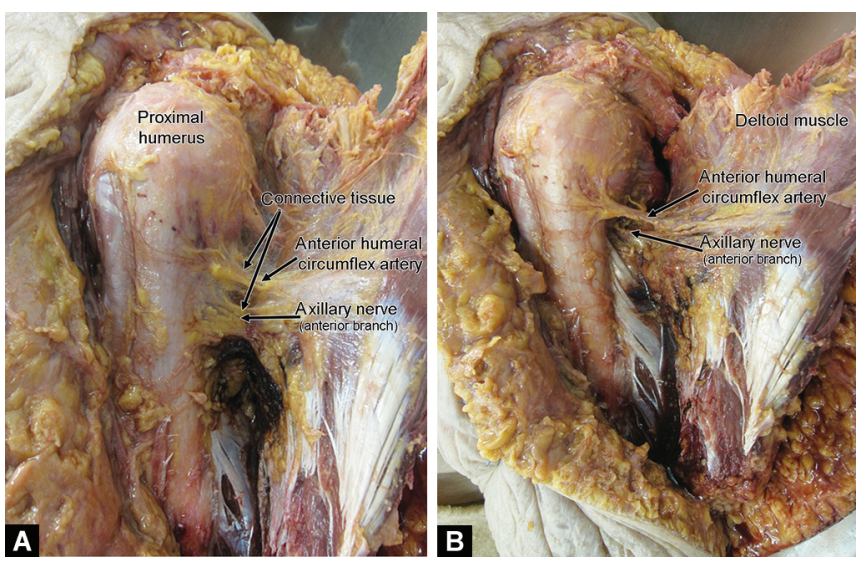

Figs $5 \mathrm{~A}$ and $\mathrm{B}$ : Connective tissue around the axillary nerve and anterior circumflex artery on the posterolateral side of the humerus. In Figure B, the connective tissue has been removed
The distal (motor) branches of the axillary nerve are also "fixed" to the deltoid muscle at the point where the inserts the muscle. So when a force directed downward from the acromion is applied, the distal end branches of the axillary nerve move along with the deltoid muscle.

To the best of our knowledge, this is the first study that takes into account the possibility of individual variability in the subacromial space, and correlates the position of the anterior branch of the axillary nerve to the deltoid muscle. We have also shown that, while applying a lateral surgical approach, the danger zone for potential nerve damage is much larger than previously thought. We therefore suggest that using the cranial top of the humeral head instead of the acromion could provide a more accurate representation of the position of the axillary nerve in dynamic clinical situations in which the subacromial space varies. We have emphasised the potential risk of axillary nerve damage during surgical procedures, and defined a zone that is relatively safe for static situations. Our preliminary results regarding the dynamic position of the nerve indicate that a safe zone for the axillary nerve in the proximal humerus may in fact be impossible to pin down.

\section{Limitations of this Study}

Since our anatomical results were restricted to specimens of Caucasian origin, they cannot by directly extrapolated to other ethnic groups.

In our study, rather than reproducing a proximal humerus fracture with osteotomies, we chose to analyse intact humerii. Due to the use of cadaver specimens, it is not clear how the amputation and the missing tension of the muscles of the shoulder affects the tension or the position of the axillary nerve. Although we consider our results highly relevant to the clinic, they cannot therefore be directly extrapolated to surgery for proximal humerus fractures.

Nevertheless, the fact that we mimicked the situation after anatomical reduction of a proximal humerus fracture meant there was no risk of misalignment, which would have changed the relationship between the nerve and bony landmarks.

\section{Future Perspectives}

We would like to study the dynamic position of the axillary nerve and its motor branches in more detail in a larger experiment using more cadavers. This would support our conclusions of the pilot experiment in which the axillary nerve appeared to move up and down around a fixed axis point. It would hopefully also provide evidence for the notion that the position of the nerve is more variable at the distal part where it runs into the deltoid muscle.

\section{Compliance with Ethical Standards}

All procedures involving human anatomical specimens were performed in accordance with the ethical standards of the institutional and/or national research committee. All of the human anatomical specimens used for our experiments had been donated for scientific research and medical training at our faculty and were part of a national body donation program approved by Dutch law and regulations.

\section{ACKnowledgments}

We, the authors, would like to thank all the people who donated their body for science and their relatives at our institution making it possible to perform this study. Special thanks go out for Ms Yvonne Steinvoort (prosector) and the Erasmus Anatomy Research Project (EARP) team for their time and effort during the practical 
sessions. Peter Frederik (PhD) and Heinrich Janzing (PhD) for their writing assistance.

\section{References}

1. Court-Brown CM, Caesar B. Epidemiology of adult fractures: a review. Injury 2006;37(8):691-697. DOI: 10.1016/j.injury.2006.04.130.

2. Rangan $A$, Handoll $H$, Brealey $S$, et al. Surgical vs nonsurgical treatment of adults with displaced fractures of the proximal humerus: the PROFHER randomized clinical trial. JAMA 2015;313(10):1037-1047. DOI: 10.1001/jama.2015.1629.

3. Mahabier KC, Vogels LM, Punt BJ, et al. Humeral shaft fractures: retrospective results of non-operative and operative treatment of 186 patients. Injury 2013;44(4):427-430. DOI: 10.1016/j.injury.2012.08.003.

4. Nicandri GT, Trumble TE, Warme WJ. Lessons learned from a case of proximal humeral locked plating gone awry. J Orthop Trauma 2009;23(8):607-611. DOI: 10.1097/BOT.0b013e3181a16100.

5. Stern R. Re: lessons learned from a case of proximal humeral locked plating gone awry. J Orthop Trauma 2010;24(1):59. DOI: 10.1097/ BOT.0b013e3181c47edfauthor reply 60.

6. Apaydin N, Tubbs RS, Loukas M, et al. Review of the surgical anatomy of the axillary nerve and the anatomic basis of its iatrogenic and traumatic injury. Surg Radiol Anat 2009;32(3):193-201. DOI: 10.1007/ s00276-009-0594-8.

7. Aszmann OC, Dellon AL. The internal topography of the axillary nerve: an anatomic and histologic study as it relates to microsurgery. J Reconstr Microsurg 1996;12(06):359-363. DOI: 10.1055/s-20071006498.

8. Bono CM, Grossman MG, Hochwald N, et al. Radial and axillary nerves. Anatomic considerations for humeral fixation. Clin Orthop Relat Res 2000. 259-264. DOI: 10.1097/00003086-200004000-00032.

9. Albritton MJ, Barnes CJ, Basamania CJ, et al. Relationship of the axillary nerve to the proximal screws of a flexible humeral nail system: an anatomic study. J Orthop Trauma 2003;17(6):411-414. DOI: 10.1097/00005131-200307000-00004.

10. Apaydin N, Uz A, Bozkurt M, et al. The anatomic relationships of the axillary nerve and surgical landmarks for its localization from the anterior aspect of the shoulder. Clin Anat 2007;20(3):273-277. DOI: 10.1002/ca.20361.

11. Kontakis GM. Is there a safe area for the axillary nerve in the deltoid muscle? J Bone Joint Surg Am 2007;89(5):1134-1135. DOI: 10.2106/00004623-200705000-00036author reply 5.

12. Ball CM, Steger T, Galatz LM, et al. The posterior branch of the axillary nerve: an anatomic study. J Bone Joint Surg Am 2003;85-A(8): 1497-1501. DOI: 10.2106/00004623-200308000-00010.

13. Uz A, Apaydin N, Bozkurt M, et al. The anatomic branch pattern of the axillary nerve. J Shoulder Elbow Surg 2007;16(2):240-244. DOI: 10.1016/j.jse.2006.05.003.

14. Loukas M, Grabska J, Tubbs RS, et al. Mapping the axillary nerve within the deltoid muscle. Surg Radiol Anat 2009;31(1):43-47. DOI: 10.1007/ s00276-008-0409-3.
15. Klitsie PJ, Ten Brinke B, Timman R, et al. Training for endoscopic surgical procedures should be performed in the dissection room: a randomized study. Surg Endosc 2017;31(4):1754-1759. DOI: 10.1007/ s00464-016-5168-3.

16. Slieker JC, Theeuwes HP, van Rooijen GL, et al. Training in laparoscopic colorectal surgery: a new educational model using specially embalmed human anatomical specimen. Surg Endosc 2012;26(8):2189-2194. DOI: 10.1007/s00464-012-2158-y.

17. Theeuwes MvR HP, Lange JF, Kleinrensink GJ. A new model for training on human specimens in surgical-anatomical skills labs. Anatomy Physiol Biochem Int J 2017;3(1):5. DOI: 10.19080/APBIJ.2017.03.555604.

18. Williams PWL. Gray's Anatomy. Edinburgh: Churchill Livingstone; 1980.

19. Sakoma $Y$, Sano $H$, Shinozaki N, et al. Anatomical and functional segments of the deltoid muscle. J Anat 2011;218(2):185-190. DOI: 10.1111/j.1469-7580.2010.01325.x.

20. Huri G, Bicer OS, Mirioglu A, et al. Humeral surface anatomy and percutaneous plate advancement: a cadaveric study. Acta Orthop Traumatol Turc 2014;48(5):584-589. DOI: 10.3944/AOTT.2014.3161.

21. Leechavengvongs $S$, Teerawutthichaikit T, Witoonchart K, et al. Surgical anatomy of the axillary nerve branches to the deltoid muscle. Clin Anat 2015;28(1):118-122. DOI: 10.1002/ca.22352.

22. de Sèze MP, Rezzouk J, de Sèze $M$, et al. Does the motor branch of the long head of the triceps brachii arise from the radial nerve? an anatomic and electromyographic study. Surg Radiol Anat 2004;26(6):459-461. DOI: 10.1007/s00276-004-0253-z.

23. Tubbs RS, Oakes WJ, Blount JP, et al. Surgical landmarks for the proximal portion of the axillary nerve. J Neurosurg 2001;95(6): 998-1000. DOI: 10.3171/jns.2001.95.6.0998.

24. Rasband WS, Image J. U. S. National Institutes of Health. Maryland, USA: Bethesda; 1997-2009.

25. Smith J, Berry G, Laflamme Y, et al. Percutaneous insertion of a proximal humeral locking plate: an anatomic study. Injury 2007;38(2):206-211. DOI: 10.1016/j.injury.2006.08.025.

26. Saran N, Bergeron SG, Benoit B, et al. Risk of axillary nerve injury during percutaneous proximal humerus locking plate insertion using an external aiming guide. Injury 2010;41(10):1037-1040. DOI: 10.1016/j. injury.2010.04.014.

27. Nijs S, Sermon A, Broos P. Intramedullary fixation of proximal humerus fractures: do locking bolts endanger the axillary nerve or the ascending branch of the anterior circumflex artery? A cadaveric study. Patient Saf Surg 2008;2(1):33. DOI: 10.1186/1754-9493-2-33.

28. Vathana $P, C$ iarapattanakom $P$, Ratanalaka R, et al. The relationship of the axillary nerve and the acromion. J Med Assoc Thai 1998;81: 953-957.

29. Schippinger G, Bailey D, McNally EG, et al. Anatomy of the normal acromion investigated using MRI. Langenbecks Arch Chir 1997;382(3):141-144. DOI: 10.1007/BF02498666.

30. Cheung S, Fitzpatrick M, Lee TQ. Effects of shoulder position on axillary nerve positions during the split lateral deltoid approach. J Shoulder Elbow Surg 2009;18(5):748-755. DOI: 10.1016/j.jse.2008.12.001. 